\title{
BMJ Open Protocol for the RT Prepare Trial: a multiple-baseline study of radiation therapists delivering education and support to women with breast cancer who are referred for radiotherapy
}

\author{
Georgia Halkett, ${ }^{1}$ Moira O'Connor, ${ }^{2}$ Sanchia Aranda, ${ }^{3,4,5}$ Michael Jefford, ${ }^{4,5}$ \\ Nigel Spry, ${ }^{6,7}$ Therese Shaw, ${ }^{8}$ Rachael Moorin, ${ }^{9}$ Penelope Schofield ${ }^{4,5}$
}

To cite: Halkett G, O'Connor M, Aranda S, et al. Protocol for the RT Prepare Trial: a multiple-baseline study of radiation therapists delivering education and support to women with breast cancer who are referred for radiotherapy. BMJ Open 2014:4:e006116. doi:10.1136/bmjopen-2014006116

- Prepublication history for this paper is available online. To view these files please visit the journal online (http://dx.doi.org/10.1136/ bmjopen-2014-006116).

Received 14 July 2014 Accepted 17 July 2014

\section{CrossMark}

For numbered affiliations see end of article.

Correspondence to Dr Georgia Halkett; g.halkett@curtin.edu.au

\section{ABSTRACT}

Introduction: There is limited evidence to guide the preparation of patients for radiotherapy. This paper describes the protocol for an evaluation of a radiation therapist led education intervention delivered to patients with breast cancer in order to reduce psychological distress.

Methods: A multiple-baseline study is being used. Usual care data is being collected prior to the start of the intervention at each of three sites. The intervention is delivered by radiation therapists consulting with patients prior to their treatment planning and on the first day of treatment. The intervention focuses on providing sensory and procedural information to patients and reducing pretreatment anxiety.

Recruitment is occurring in three states in Australia. Eligible participants are patients who have been referred for radiotherapy to treat breast cancer. 200 patients will be recruited during a usual care phase and, thereafter, 200 patients in the intervention phase. Measures will be collected on four occasionsafter meeting with their radiation oncologist, prior to treatment planning, on the first day of treatment and after treatment completion. The primary hypothesis is that patients who receive the radiotherapy preparatory intervention will report a significantly greater decrease in psychological distress from baseline to prior to radiotherapy treatment planning in comparison with the usual care group. Secondary outcome measures include concerns about radiotherapy, patient knowledge of radiotherapy, patient preparedness and quality of life. Patient health system usage and costs will also be measured. Multilevel mixed effects regression models will be applied to test for intervention effects.

Ethics: Ethics approval has been gained from Curtin University and the three recruiting sites.

Dissemination: Results will be reported in international peer reviewed journals.

Trial registration number: Australian and New Zealand Clinical Trials Registration: ACTRN12611001000998.

\section{Strengths and limitations of this study}

- This is the first multiple-baseline study testing the effectiveness of an innovative radiotherapy preparatory intervention to reduce patient psychological distress before treatment.

- This study is based on our pilot work and in being tested at three sites across Australia.

- The cost effectiveness of this intervention will also be determined.

\section{INTRODUCTION}

Worldwide, breast cancer is the most common cancer affecting women. ${ }^{1}$ Evidencebased recommendations suggest that approximately $48 \%$ of all people diagnosed with cancer and $83 \%$ of all people diagnosed with breast cancer should receive radiotherapy at some stage during their illness. ${ }^{2}{ }^{3}$ However, the average radiotherapy utilisation rate in Australia for all cancers is $38 \%$ and at least 18000 patients did not receive potentially beneficial radiotherapy in $2012 .{ }^{4}$ Inadequate referral, lack of access to treatment facilities and refusal of treatment by patients have been attributed to reasons for patients not receiving radiotherapy. ${ }^{2}$ Patients may decline treatment because they lack knowledge of radiotherapy ${ }^{5-8}$ and fear treatment and its associated side effects. ${ }^{9}$

Information and support needs of breast cancer patients

D'Haese $e t a l^{10}$ reported that patients prefer to receive information staggered over time. Halkett et $a l^{11}$ mapped the specific radiotherapy-related information that patients desire and demonstrated that information needs peak at the time of treatment planning and 
again prior to treatment. Patients express a need for information about procedures, their role, any pain or discomfort they might experience and side effects.

Also, there is growing evidence to suggest that the psychosocial needs of patients with cancer are not adequately met $^{12}$ and that psychological morbidity is underrecognised and undertreated, despite effective treatments being available. ${ }^{13}$ Untreated comorbid psychological conditions can be detrimental to patients in terms of pain and physical functioning ${ }^{14}$ and lead to higher medical costs and longer hospital stays. ${ }^{15}$ Halkett $e t a l^{16}$ conducted a longitudinal study of patients with breast cancer as they proceeded from referral to radiotherapy until after treatment completion and found that prior to treatment planning $30 \%(37 / 123)$ of women had clinically relevant levels of anxiety ${ }^{17}$ and $10 \%$ had clinically relevant levels of depression. After treatment planning, 26\% of patients continued to have clinical levels of anxiety and $10 \%$ had clinical levels of depression. After starting treatment anxiety levels did not change.

Inadequate communication and information provision increase patient anxiety, reduce the patient's sense of control and confidence in health professionals and, in some cases, lead to people declining treatment that might otherwise improve their chance of survival. ${ }^{18}$ Whereas, sufficient and timely information provision increases patient satisfaction and reduces psychological distress. $^{19}$

\section{Roles of health professionals in radiotherapy}

Information provision within radiotherapy departments is inconsistent in terms of who provides information and how and when information is provided. ${ }^{20}$ Radiation oncologists initially consult patients and provide education. Radiation oncology nurses guide patients in managing side effects and play a role in providing education. Radiation therapists also provide information and support during treatment. ${ }^{21}$ However, the most appropriate education strategy prior to radiation therapy remains unclear. Standardised procedures are not followed by radiation therapists who provide information and it may be delivered when the patient is on the radiotherapy treatment table during treatment planning or treatment. ${ }^{20}$ This method of information delivery is not the optimum way to deliver education because patients are unlikely to recall the information provided and it does not allow radiation therapists to address pretreatment anxiety.

\section{Preparing patients for potentially threatening procedures} such as radiotherapy

A recent systematic review on interventions for preparing patients for chemotherapy and radiotherapy highlighted the need for rigorous studies to provide further evidence to guide the preparation of patients for radiotherapy. ${ }^{22}$ Although a number of researchers have developed information resources (eg, videos and written information) for patients who require radiotherapy, ${ }^{23-26}$ the aim of these studies has been to provide general information about radiotherapy and side effects rather than specific information on what to expect at each stage and focusing on addressing patients' anxiety. A significant reduction in anxiety was found in one study after providing patients with an educational video; however, this reduction in anxiety was only recorded after the treatment began rather than at the time of radiotherapy treatment planning or prior to start of the treatment. ${ }^{24}$ The anxiety levels and fear that patients experience prior to radiotherapy indicate that it is perceived to be a threatening procedure. This suggests that radiotherapy education might best be approached using Level I evidence on preparing patients for threatening medical procedures from other areas of health. This evidence suggests that preparation for threatening medical procedures should include: (1) sensory and procedural information and (2) techniques for addressing treatment related concerns and anxiety. ${ }^{27-30}$ Sensory information relates to informing patients about how they are likely to feel during treatment planning and treatment; procedural information relates to describing the procedure they will undergo. Research demonstrates that preparation comprising these components assists in improving health outcomes and is more effective in reducing patient anxiety levels than other interventions. ${ }^{27-30}$ Aranda $e t a l^{31}$ found that when an educational intervention, consisting of sensory and procedural information and anxiety reduction strategies was provided to chemotherapy patients using a randomised controlled trial, patients in the intervention group had significantly lower sensory/psychological $(\mathrm{p}=0.027)$ and procedural $(\mathrm{p}=0.03)$ concerns after starting chemotherapy. Patients who had elevated levels of distress and received the intervention also indicated a significant decrease in distress at this time $(\mathrm{p}=0.035)$.

Currently, in some radiation oncology sites in Australia, radiation therapists are not involved in providing patients with information until they are already on the treatment table. ${ }^{20}$ During our observations of usual care we found that information provided does not comply with the literature on preparing patients for threatening procedures, very little sensory or procedural information is given (if at all) and radiation therapists do not spend time eliciting or addressing patients' fears and concerns. ${ }^{32}$ Furthermore, radiation therapists often do not have the opportunity to follow patients up when they start treatment or provide continuity of care. Applying this evidence to a radiotherapy population is necessary to determine whether the introduction of such an educational intervention, which focuses on procedural and sensory information and addresses treatment related anxiety, similarly reduces patients' levels of psychological distress.

\section{Aims of the Radiation Therapy Prepare Trial}

The Radiation Therapy (RT) Prepare Trial aims to examine the effectiveness of an innovative radiotherapy 
preparatory intervention, consisting of two one-on-one educational sessions, delivered by radiation therapists to: (1) reduce patient psychological distress before treatment; (2) reduce specific concerns about radiotherapy; (3) increase patient knowledge of radiotherapy and (4) increase patient preparedness for their treatment planning appointment and treatment using a multiplebaseline design trial. The cost effectiveness of this intervention will also be determined.

\section{Primary hypothesis}

Patients who receive the radiotherapy preparatory intervention will report a significantly greater decrease in psychological distress from baseline to the first follow-up data collection (conducted prior to radiotherapy treatment planning), in comparison with the usual care group.

\section{Secondary hypotheses}

1. Patients in the intervention group will report a significantly greater decrease in psychological distress in comparison with the usual care group from baseline to the second follow-up data collection (conducted prior to the first treatment).

2. Patients in the intervention group will report a significantly greater reduction in concerns about radiotherapy and a significantly greater increase in knowledge of radiotherapy and preparedness for their treatment planning appointment and treatment compared with patients in the usual care group from baseline to (A) follow-up 1 and (B) follow-up 2.

3. Patients in the intervention group will experience better outcomes, as measured by the incremental cost per quality adjusted life years (QALY), during and after treatment compared with the patients in the usual care group.

4. Patients in the intervention group will utilise less healthcare resources compared with patients in the usual care group from baseline to (A) follow-up 1 and (B) follow-up 2.

\section{Pilot study}

In 2008, the multidisciplinary project team began developing the proposed intervention to address the information needs identified and reduce psychological distress. The team included representatives from radiation oncology, behavioural science, clinical psychology and psychiatry, general practice, nursing and a consumer. Ten radiation therapists were trained in delivering the intervention package. Postworkshop evaluation surveys showed that radiation therapists were highly satisfied and found that these workshops assisted them to deliver the education sessions effectively. The pilot study demonstrated that this intervention is clinically feasible and acceptable to health professionals and patients. ${ }^{33}$ For our pilot randomised controlled study, we recruited 122 participants at a single site. ${ }^{34}$ Sixty-four participants received the intervention and 58 received usual care.
After the first intervention, at the time of CT planning patient anxiety was significantly lower in the intervention group $(\mathrm{p}=0.048)$. Patients concerns about radiotherapy were also significantly reduced $(\mathrm{p}=0.001)$. Patient knowledge in the intervention group was also higher at the time of CT planning $(\mathrm{p}<0.001) .^{34}$ These findings support moving the intervention into a larger and adequately powered study.

\section{METHODS}

\section{Trial design}

A multiple-baseline methodology (time series design) is being used to trial this intervention. Multiple-baseline designs are advocated when undertaking health services research interventions that seek to change clinician behaviour. ${ }^{35}$ This method will demonstrate whether (1) a change in psychological distress and knowledge has occurred, (2) the change is a likely result of the intervention and (3) the change is statistically significant and clinically relevant. ${ }^{35}$ This methodology facilitates a systematic comparison of preintervention and postintervention measures while controlling for other factors ${ }^{36}$ and the outcome measures can be measured reliably on repeated occasions. ${ }^{37} 38$ The use of this design will enable us to be more confident that the introduction of the intervention is responsible for change in the outcomes measures in the time series if there are multiple time series, each of which receives the intervention at a different point in time. ${ }^{37}$ This methodology is more appropriate, cost effective and practical than a cluster randomised controlled trial because usual care varies between the recruitment sites (negating the use of some sites as control sites only). A randomised controlled trial is also not appropriate because staff work across multiple sites and contamination could occur when staff who have been trained to deliver the intervention are allocated to treat a usual care patient. ${ }^{35} 39$ This multiplebaseline design will detect whether the intervention is effective in one setting, but not in another (eg, it may be dependent on how departments are managed or which models of service delivery are used). ${ }^{37}$

All sites are initially collecting data at all measurement points from patients who are receiving usual care as they proceed through treatment. This data collection before intervention implementation will provide a 'baseline' description of the underlying trends, that is, changes in the outcome measures during the course of a patient's treatment without the proposed intervention and any departmental and cyclical (eg, seasonal) effects to be estimated. ${ }^{40}$ Rather than randomising individual patients to the intervention phase, sites were randomly selected to begin the intervention at different time points after usual care data collection. The data collected after introduction of the intervention will allow for the intervention effect to be assessed, while accounting for any underlying secular trends, ${ }^{40}$ that is, the extent to which the intervention impacts on the changes in the outcome 
measures during the treatment period over and above what occurs during usual care.

The TREND (Transparent Reporting of Evaluations with Non-Randomised Designs) guidelines are being followed to ensure the following information is recorded: theories used and descriptions of intervention and comparison conditions; research design; and methods of adjusting for possible bias. ${ }^{41}$

Figure 1 provides an understanding of the timing of intervention delivery and survey distribution. Figure 2 demonstrates how we are using the multiple-baseline method and when each site starts the intervention. Figure 3 provides a diagrammatic representation of the survey instruments, timing of intervention delivery and the content of intervention delivery.

\section{Intervention content}

The tailored educational intervention package consists of two individual consultations with a radiation therapist (1) prior to treatment planning and (2) prior to first day of treatment (figure 1). The intervention focuses on radiation therapists providing sensory and procedural information, and using anxiety reduction strategies with patients. During the first consultation, prior to treatment planning, radiation therapists identify patients' fears and anxiety and discuss topics related to the treatment planning procedure centring around the information patients require and any concerns they identify. ${ }^{33}{ }^{34}$ During the second consultation, prior to treatment, radiation therapists again identify patients' fears and anxiety and discuss information needs relating to treatment initiation and what to expect. ${ }^{33}$ Each patient was asked to attend their treatment planning or treatment $30 \mathrm{~min}-1 \mathrm{hr}$ early to allow time for the intervention to be delivered.

Strategies that are being used to detect anxiety and distress include: active listening and using open questions, responding to patients' emotional cues and adopting a patient-centred communication style. ${ }^{42}$ If patients are experiencing anxiety or distress, the radiation therapist discusses how they are feeling and coping methods, and the patient may also be referred for psychological support if required. These techniques are standardised across sites and evidence-based literature describing these techniques are included in the study manual.

\section{Settings}

Participating sites are located in the capital cities of three Australian states, Perth, Adelaide and Melbourne. The sites were chosen because they are of varying sizes and in different socioeconomic settings. Recruitment started from the end of July 2012 and will be ceased at the end of May 2015.

\section{Sample recruitment}

A consecutive sample is being recruited from each site within each of the control (usual care) and intervention phases. Women with breast cancer referred for radiotherapy are being recruited. Eligibility criteria: diagnosed with breast cancer and referred for a curative course of radiotherapy ( $>50 \mathrm{~Gy}$ equivalent), have not started treatment or participated in a treatment planning appointment, the treatment planning appointment is scheduled at least 2 days after recruitment, do not have cognitive impairments or psychiatric illnesses and are able to communicate in English.

\section{Recruitment procedures}

Patients are recruited following their first consultation with their radiation oncologist. After the patient is identified by the radiation oncologist, the research assistant assesses patient eligibility criteria and invites patients to participate. All patients who meet the eligibility criteria and attend the sites within the study period are approached. Women who are receiving sequential chemotherapy and radiotherapy as well as those who have been referred for radiotherapy alone are included.

\section{Randomisation}

Sites were randomly allocated to intervention initiation dates. The staggered switch from usual care to the intervention phase is designed to facilitate comparisons between the phases and ensure that any observed changes in the outcome measures are not due to extraneous influences that may occur at a particular point in time. ${ }^{35}$

\section{Measures}

The primary outcome measure is psychological distress measured using the Hospital Anxiety and Depression Scale (HADS). ${ }^{17}$ The secondary outcome measures are (1) psychological distress measured using the HADS; (2) specific concerns about radiotherapy measured by the Concerns about RT scale; ${ }^{43}$ (3) patient knowledge of radiotherapy measured by the Knowledge of RT scale; (4) patient preparedness measured by the Cancer Treatment Survey; ${ }^{44}$ (5) Assessment of Quality of Life Survey (AQoL-6D) ${ }^{45}$ and (6) Patient Health System

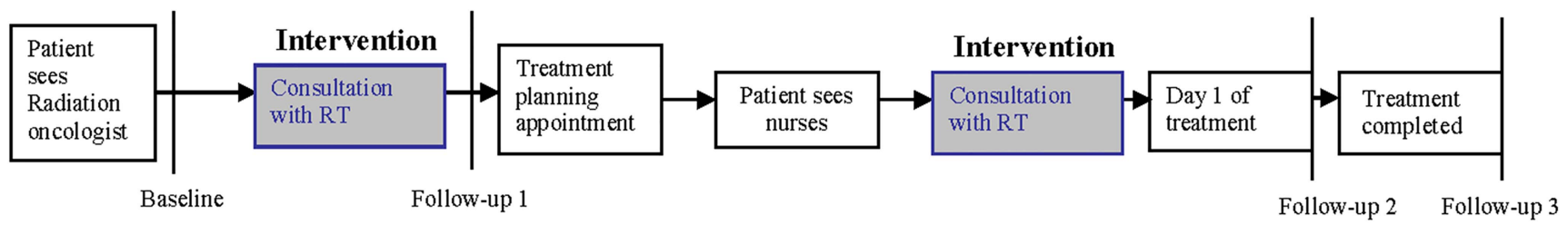

Figure 1 Timing of intervention delivery and survey distribution (RT, radiation therapist). 


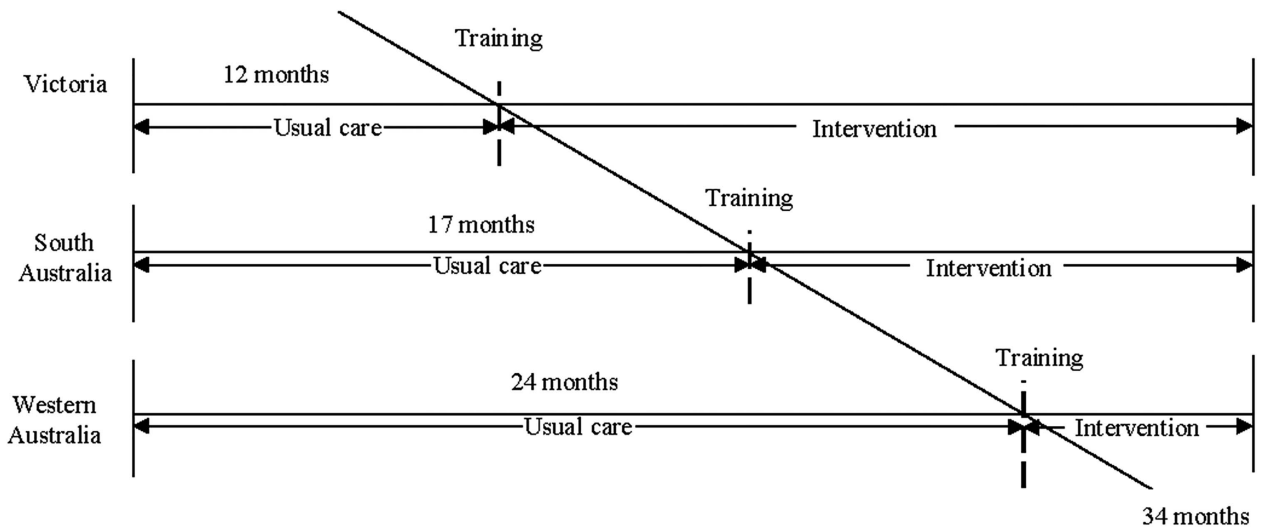

Figure 2 Trial design.

Usage and Cost Questionnaire (PHSUCQ). Each of these measures is described below.

\section{Hospital Anxiety and Depression Scale (HADS)}

This scale is being used to measure psychological distress. It contains 14 items; seven items measure Anxiety (HADS-A) and seven measure Depression (HADS-D). These scores are combined to determine a total score (HADS-T). The HADS-T is well-established as a general measure of emotional distress in patients with cancer ${ }^{46}$ and our previous work demonstrated that it is responsive to educational interventions. ${ }^{47}$ A HADS-T cut-off of greater than or equal to 15 is indicative of high anxiety and/or depression.

\section{Concerns about RT scale (C-RT)}

This nine-item scale asks patients to identify how concerned they feel about specific aspects of radiotherapy (eg, work, side effects, equipment, impact on life and future) using a nine-point Likert scale. This scale has high internal consistency, achieving a Cronbach's $\alpha$ of 0.91 , and adequate stability over a 10-day time period (mean Intraclass Correlation Coefficient (ICC) $=0.60$, $\mathrm{SD}=0.097) .{ }^{43}$ Following reliability testing of this scale, it has been used in a further study with 120 patients and achieved a Cronbach's $\alpha$ of $0.86 .{ }^{16}$

\section{Knowledge of RT scale (K-RT)}

This scale asks patients to identify their current level of knowledge about different aspects of radiotherapy using a Likert scale. It is based on The RT Information Needs Scale previously developed by Halkett $e t a l,{ }^{43}$ which has high internal consistency (Cronbach's $\alpha$ of 0.86 ) and adequate stability over time (mean ICC $=0.55, \mathrm{SD}=0.18$ ). Following reliability testing, it has been used in a further study and achieved a Cronbach's $\alpha$ of $0.86 .{ }^{16}$

\section{Cancer Treatment Survey (CaTS)}

This scale on patient preparedness for cancer treatment was developed and tested by Schofield $e t a t^{4}$ and is theoretically based on the evidence for preparing patients for threatening medical procedures. It contains 25 items that assess sensory/physiological and procedural concerns. The subscales have high internal consistency (Cronbach's $\alpha>0.90$ ) and the average interitem correlation for each subscale exceeds $0.3{ }^{44}$ This scale was also sensitive to change following delivery of an educational intervention in the chemotherapy setting. ${ }^{31}$

\section{Assessment of Quality of Life survey (AQoL-6D)}

This scale is a reliable and valid measure of health status for clinical and economic appraisal. ${ }^{45}{ }^{48}$ It consists of 20 questions relating to independent living, relationships, mental health, coping and pain. A unique feature of the AQoL-6D is that the utility weights have been derived from an Australian population sample. Norms for the Australian population are available for this questionnaire. ${ }^{49}$

PHSUCQ: This is a purpose built questionnaire that assesses the use of services within the health system and their associated cost to the patient. It also collects information about the use of medicines and complementary practices such as reflexology and acupuncture. Participants are also asked to identify whether they have private health insurance and whether they needed to take leave from work. Responses are open ended throughout this questionnaire.

\section{Intervention costs}

Additional costs are associated with the intervention programme. The main cost is staff time due to training and the one-on-one consultations with a radiation therapist prior to treatment planning and the first day of treatment. The costs will be quantified using the hourly salary of the staff involved multiplied by the time taken for training (one off cost for each radiation therapist providing the intervention) and the two consultations for each patient.

\section{Training for radiation therapists}

Radiation therapists will be responsible for delivery of the intervention. For each site, a training programme will be implemented by trained communication skills educators following completion of the collection of data from usual 


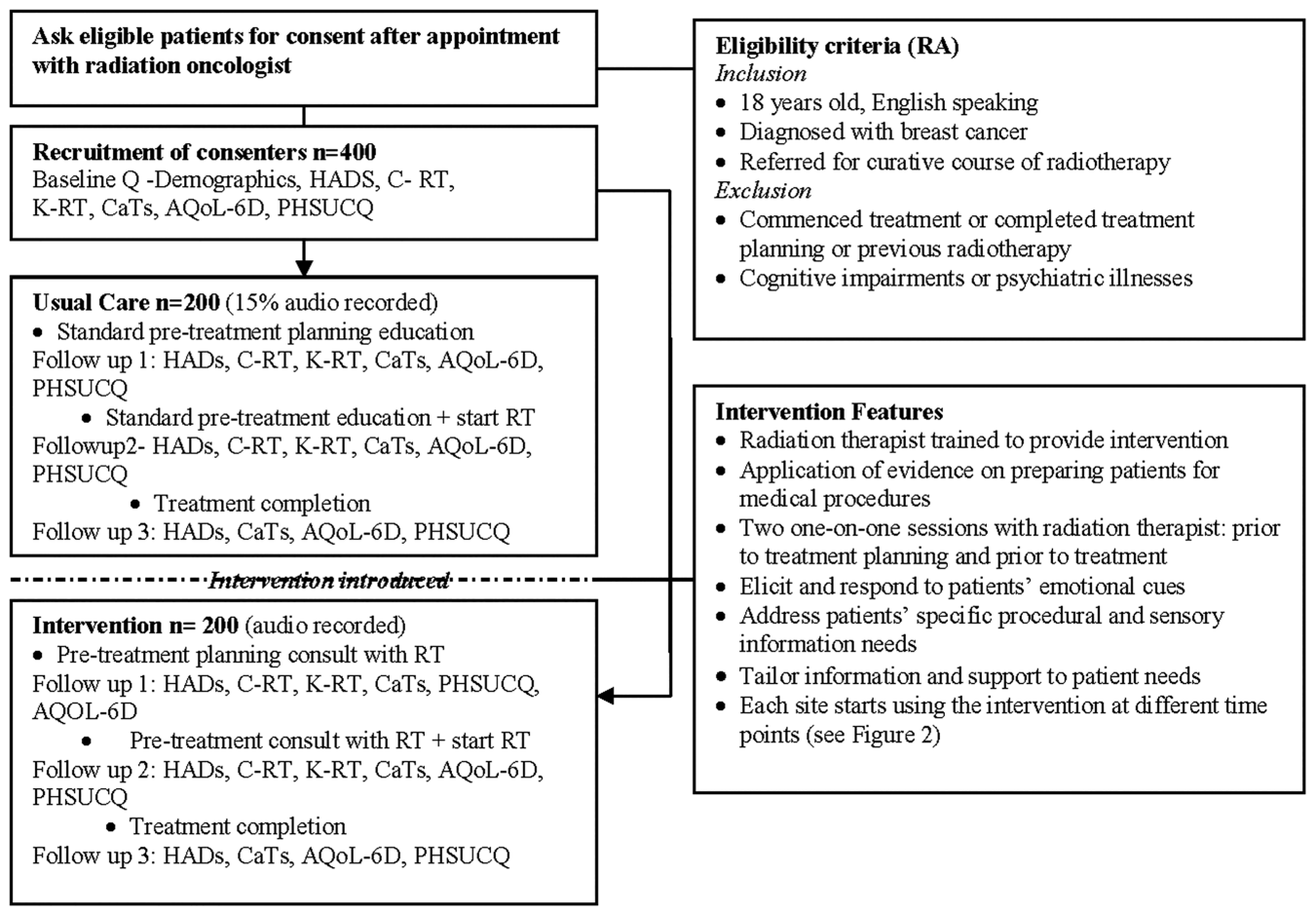

Figure 3 Study plan (AQoL-6D, Assessment of Quality of Life survey; CaTS, Cancer Treatment Survey; C-RT, Concerns about RT scale; HADS, Hospital Anxiety and Depression Scale (HADS); K-RT, Knowledge of RT scale; PHSUCQ, Patient Health System Usage and Cost Questionnaire; Q, questionnaire; RA, research assistant).

care patients. The training consists of: (1) a communication skills workshop focused on eliciting and responding to emotional cues; (2) a radiotherapy specific workshop focusing on radiotherapy sensory and preparatory information and (3) a study manual outlining evidence based strategies for dealing with patient concerns.

\section{Communication skills training}

A recent systematic review of communication skills training programmes found that these programmes improve health professionals' communication skills in terms of conducting psychological assessment, interviewing skills, attitudes towards communicating with patients and health professionals' confidence in their ability to communicate. ${ }^{50}$ The eliciting and responding to emotional cues workshop ${ }^{51}$ will be used because this assists health professionals to detect patients' emotions and respond to them appropriately. During the workshop, radiation therapists have the opportunity to practice communicating with simulated patients (actors) who display different emotions and concerns. Radiation therapists are then provided with feedback by the facilitator, actor and peers.

\section{Radiotherapy-specific workshop}

This workshop focuses on training radiation therapists to prepare patients for treatment planning and treatment. During the workshop we provide radiation therapists with an outline of published evidence and outline the information needs that are highest for patients and how these information needs can be best addressed. ${ }^{16}$ We then use an adapted version of Pendleton's ${ }^{52}$ model of patient consultations to guide radiation therapists in relation to tasks they need to perform. We also provide a checklist that radiation therapists can use during consultations and give them the opportunity to practice their skills with a trained actor.

\section{Study manual}

An evidence based study manual has been compiled for radiation therapists to use as a guide. Within this manual we provide evidence-based literature ${ }^{29} \quad 42 \quad 53$ about communicating and the procedural and sensory information patients commonly desire. ${ }^{11}{ }^{16}$ We also provide a checklist that guides radiation therapists as they proceed through each of the consultations with the patients.

\section{Quality assurance of the intervention}

For each intervention consultation a checklist completed by the radiation therapist will assess adherence to the protocol and the radiation therapist's perceptions of the delivery, including the environment, interruptions, people present and completeness of information. All treatment planning and treatment consultations conducted for the intervention will be digitally recorded. Recordings of the first 10 intervention sessions for each site will be reviewed by the research team for compliance. Following this, $15 \%$ of all other recordings are being randomly selected and analysed by two trained reviewers to ensure the intervention is delivered as per the protocol. During the pilot study, a protocol for analysing these tape recordings was developed and trialled 
by two independent staff members. ${ }^{31}$ Radiation therapists participating in intervention delivery are provided with routine feedback about the consultations during follow-up workshops and invited to participate in interviews about delivering the intervention.

\section{Documenting usual care}

Usual care prior to the start of radiotherapy varies between sites. Research assistants in each of the sites tape record $15 \%$ of usual care appointments with RTs in order to document the usual care that is provided. The research assistant informs the patient and RT (with their consent) to obtain a recording of what information is provided to patients. The research assistant documents, using a checklist, the content of the information provided.

\section{Sample size requirements and power calculation}

Using a 5\% significance level two-tailed testing of differences between two independent groups (usual care and intervention), a sample of 100 patients from the intervention and 100 patients from the control phase of the study would have $80 \%$ power to detect group differences between patients under these two experimental conditions of $0.40 \mathrm{SDs}$ for the primary outcome measure. ${ }^{54}$ This equates to 200 patients in total, 67 per site. Adjusting for a design effect of 1.66 (assuming an ICC $=0.01$ and 67 patients for each of the 3 sites), the required sample size is a total of 332 patients (166 in each of the two phases and 111 per site). Allowing for attrition of $20 \%$ from baseline to the second follow-up, 400 patients need to be recruited to the study at baseline, that is, 200 to each of the usual care and the intervention phase, respectively.

From our pilot data, a difference in reduction of psychological distress using HADS-T was at least 0.66 $(\mathrm{SD}=2)$ between the usual care and intervention groups, suggesting an effect size in the order of 0.4 is appropriate. While the study is conservatively powered to detect a small-moderate effect size of 0.4 , it is hoped that the intervention will result in moderate (0.5) or larger effects. $^{54}$

\section{Data analysis}

Non-parametric statistics will be used when appropriate. Recruitment bias will be assessed by comparing available demographic and clinical variables for participants with those who decline participation using $t$ tests or Mann-Whitney $U$ tests for continuous variables and $\chi^{2}$ tests for categorical variables. Possible differential attrition will be assessed by comparing baseline characteristics of those who withdraw with those who remain in the study using t tests or Mann-Whitney U tests for continuous variables and $\chi^{2}$ tests for categorical variables.

\section{Intention to treat analyses}

The effect of the intervention will be assessed by conducting multilevel mixed effects regression models (in
Stata V.12), controlling for site-level clustering and incorporating a time by group interaction to determine whether trends across the three data points within the course of the patients' treatment differ between the intervention and usual care groups. ${ }^{55}$ Multiple imputation methods will be applied to reduce the impact of missing data. The models will adjust for confounders and effect modifiers as necessary. Models will be fitted for the primary outcome measure (HADS-T) as well as the other outcome measures: specific concerns about radiotherapy, patient knowledge of radiotherapy and patient preparedness. Model assumptions, such as normality assumptions, will be tested and appropriate methods used to ensure assumptions are met.

\section{Cost-utility analysis}

This analysis will be based on comparing the cost of additional resources required for the intervention, health services utilisation costs as recorded from the patients' medical notes and possible subsequent improvement in health outcomes (measured using the AQoL-6D). Health outcomes will be assessed in terms of QALYs. The cost-utility analysis will provide an incremental costeffectiveness ratio, expressed as the cost per QALY, representing the difference in costs and quality adjusted outcomes between the two groups of patients. ${ }^{56}$

\section{Analysis of health cost offset}

Health service utilisation will be captured using the PHSUCQ. The utilisation of services can be converted into direct monetary costs by using the fees charged for services outside of hospitals and information on inpatient and outpatient treatment costs in hospitals. A comparison of the cost of utilisation of medical services for the intervention and usual care groups will provide an estimate of the impact of preparatory information and associated reduction in distress on healthcare costs.

\section{DISCUSSION}

This study adopts a multiple-baseline method to implement a methodologically rigorous trial of an innovative intervention in which radiation therapists take an active role in providing sensory and procedural information and anxiety reduction techniques using a consultation prior to treatment planning and treatment at key time points during the patient's radiotherapy journey. It involves radiation therapists detecting patients' emotional cues and communicating with patients, tailoring sensory and procedural information and introducing anxiety-reduction techniques. The effectiveness of the intervention will be assessed in terms of psychological distress, patient concerns and knowledge about radiotherapy and patient preparedness. Not only has this proposed educational package the potential to reduce psychological distress, it may also reduce the overall cost of health provision to these patients because failing to meet patients' psychological and supportive care needs 
has been demonstrated to result in additional referral for psychological care and higher medical costs. ${ }^{15}$

During this study we are measuring the health service utilisation by patients in both phases and will conduct a cost utility analysis to determine whether the patients in the intervention group experience better outcomes during and after treatment compared with the patients in the usual care group as measured by the incremental cost per QALY. If an effective, systematic approach to preparing patients about radiotherapy is shown to be successful and routinely adopted, it may also enhance patient uptake of radiotherapy, improve the patients' experience of receiving radiotherapy, improve selfreported health quality and health distress, and result in fewer hospitalisations and reduced health costs overall. ${ }^{57}$

This research has the potential to change how patients are prepared for radiotherapy and the proposed intervention enhances the role of radiation therapists in educating and supporting patients. This research would be particularly beneficial if it could be extrapolated for other cancer sites to improve: treatment compliance, patient self-efficacy about side effects and toxicity management, and management of toxicity post treatment completion. The pilot study demonstrated that the intervention is acceptable and can be integrated into clinical practice. Furthermore, during the pilot study significant changes between the time points were found for the intervention group in comparison with usual care for both preparation and knowledge, and there was also a reduction in psychological distress for intervention patients over time. ${ }^{33} 34$

\section{CONCLUSION}

We believe that this is the first study internationally to systematically test an intervention to address patients' information and support needs throughout their radiotherapy treatment trajectory and reduce patients' levels of anxiety and depression (psychological distress) prior to treatment initiation. The proposed intervention package uses an innovative approach, which optimises radiation therapists' involvement in preparing patients for radiotherapy prior to treatment planning and prior to treatment, to meet patients' information needs and reduce their levels of psychological distress.

\footnotetext{
Author affiliations

${ }^{1}$ School of Nursing and Midwifery, Faculty of Health Sciences, Curtin University, Perth, Western Australia, Australia

${ }^{2}$ School of Psychology and Speech Pathology, Faculty of Health Sciences, Curtin University, Perth, Western Australia, Australia

${ }^{3}$ Cancer Services and Information, Cancer Institute NSW, Sydney, New South Wales, Australia

${ }^{4}$ Department of Cancer Experiences Research, Peter MacCallum Cancer Centre, East Melbourne, Victoria, Australia

${ }^{5}$ Sir Peter MacCallum Department of Oncology and Faculty of Medicine, Dentistry and Health Sciences, The University of Melbourne, Melbourne, Victoria, Australia

${ }^{6}$ Department of Radiation Oncology, Sir Charles Gairdner Hospital, Perth, Western Australia, Australia
}

${ }^{7}$ Department of Medicine and Pharmacology, University of Western Australia, Perth, Western Australia, Australia

${ }^{8}$ School of Exercise and Health Science, Edith Cowan University, Perth, Western Australia, Australia

${ }^{9}$ Faculty of Health Sciences, Curtin University, Perth, Western Australia, Australia

Contributors GH, MO, SA, MJ and PS coordinated the design, grant application and implementation of the study and led the writing of the manuscript. NS provided clinical expertise and contributed to the grant application and writing of the manuscript. TS provided statistical input into the design and grant application and contributed to the writing of the manuscript. RM provided health economics advice and contributed to the grant application and writing of the manuscript.

Funding This grant \#APP1006458 was awarded through the Priority-driven Collaborative Cancer Research Scheme and funded by Cancer Australia and Beyond Blue.

\section{Competing interests None.}

Ethics approval Curtin University, Peter MacCallum Cancer Centre, Royal Adelaide Hospital, Sir Charles Gairdner Hospital.

Provenance and peer review Not commissioned; peer reviewed for ethical and funding approval prior to submission.

Open Access This is an Open Access article distributed in accordance with the Creative Commons Attribution Non Commercial (CC BY-NC 4.0) license, which permits others to distribute, remix, adapt, build upon this work noncommercially, and license their derivative works on different terms, provided the original work is properly cited and the use is non-commercial. See: http:// creativecommons.org/licenses/by-nc/4.0/

\section{REFERENCES}

1. Ferlay J, Soerjomataram I, Ervik M, et al. Cancer Incidence and Mortality Worldwide: IARC Cancer Base No. 11. GLOBOCAN 2012 v1.0. 2013.

2. Delaney G, Barton M, Jacob S. Estimation of an optimal radiotherapy utilization rate for breast carcinoma: a review of the evidence. Cancer 2003;98:1977-86.

3. Barton MB, Jacob S, Shafiq J, et al. Estimating the demand for radiotherapy from the evidence: a review of changes from 2003 to 2012. Radiother Oncol 2014. In press.

4. Radiation Oncology Tripartite Committee. Planning for the best: tripartite national strategic plan for radiation oncology 2012-2022. The Royal Australian and New Zealand College of Radiologists, 2012.

5. Gamble K. Communication and information: the experience of radiotherapy patients. Eur J Cancer Care 1998;7:153-61.

6. Long L. Being informed: undergoing radiation therapy. Cancer Nurs 2001;24:463-8.

7. Johnson J. "Living with radiotherapy": the experiences of women with breast cancer. J Radiother Pract 1999;1:17-25.

8. Martin K-L, Hodgson D. The role of counselling and communication skills: how can they enhance a patient's 'first day' experience? $J$ Radiother Pract 2006;5:157-64.

9. Halkett G, Kristjanson L, Lobb E. "If we get too close to your bones they'll go brittle": women's fears about radiotherapy for early breast cancer. Psychooncology 2008;17:877-84.

10. D'haese S, Vinh-Hung V, Bijdekerke P, et al. The effect of timing of the provision of information on anxiety and satisfaction of cancer patients receiving radiotherapy. J Cancer Educ 2000;15:223-7.

11. Halkett G, Kristjanson L, Lobb E, et al. Meeting breast cancer patients' information needs during radiotherapy: What can we do to improve the information and support that is currently provided? Eur $J$ Cancer Care 2010;19:538-47.

12. Sanson-Fisher R, Girgis A, Boyes A, et al. The unmet supportive care needs of patients with cancer. Cancer 2000;88:226-37.

13. Newell SA, Sanson-Fisher RW, Savolainen NJ. Systematic review of psychological therapies for cancer patients: overview and recommendations for future research. J Natl Cancer Inst 2002;94:558-84.

14. Katon W, Lin EH, Kroenke K. The association of depression and anxiety with medical symptom burden in patients with chronic medical illness. Gen Hosp Psychiatry 2007;29:147-55. 
15. Carlson L, Bultz B. Benefits of psychosocial oncology care: improved quality of life and medical cost offset. Health Qual Life Outcomes 2003;1:8.

16. Halkett GK, Kristjanson LJ, Lobb E, et al. Information needs and preferences of women as they proceed through radiotherapy for breast cancer. Patient Educ Couns 2012;86:396-404.

17. Zigmond AS, Snaith RP. The Hospital Anxiety and Depression Scale. Acta Psychiatr Scand 1983;67:361-70.

18. Jefford $\mathrm{M}$, Tattersall $\mathrm{MH}$. Informing and involving cancer patients in their own care. Lancet 2002;3:629-37.

19. Schofield P, Butow P, Thompson J, et al. Psychological responses of patients receiving a diagnosis of cancer. Ann Oncol 2003; $14: 48-56$.

20. Halkett G, Short M, Kristjanson L. How do radiation oncology health professionals inform breast cancer patients about the medical and technical aspects of their treatment? Radiother Oncol 2008;90:153-9.

21. Halkett G, Kristjanson L. Patients' perspectives on the role of radiation therapists. Patient Educ Couns 2007;69:76-83.

22. Waller A, Forshaw K, Bryant J, et al. Interventions for preparing patients for chemotherapy and radiotherapy: a systematic review. Support Care Cancer 2014;22:2297-308.

23. Dunn J, Steginga S, Rose P, et al. Evaluating patient education materials about radiation therapy. Patient Educ Couns 2004;52:325-32.

24. Thomas R, Daly M, Perryman B, et al. Forewarned is forearmedbenefits of preparatory information on video cassette for patients receiving chemotherapy or radiotherapy-a randomised controlled trial. Eur J Cancer 2000;36:1536-43.

25. Haggmark C, Bohman L, IImoni-Brandt K, et al. Effects of information supply on satisfaction with information and quality of life in cancer patients receiving curative radiation therapy. Patient Educ Couns 2001:45:173-9.

26. Jahraus D, Sokolosky S, Thurston N, et al. Evaluation of an education program for patients with breast cancer receiving radiation therapy. Cancer Nurs 2002;25:266-75.

27. Suls J, Wan C. Effects of sensory and procedural information on coping with stressful medical procedures and pain: a meta-analysis. J Consult Clin Psych 1989:57:372-9.

28. Kok G, van der Borne B, Mullen P. Effectiveness of health education and health promotion: meta-analysis of effect studies and determinants of effectiveness. Patient Educ Couns 1997;30:19-27.

29. National Breast Cancer Centre \& National Cancer Control Initiative. Clinical practice guidelines for the psychosocial care of adults with cancer. Camperdown, New South Wales: National Breast Cancer Centre, 2003.

30. Hathaway D. Effect of preoperative instruction on postoperative outcomes: a meta-analysis. Nurs Res 1986;35:269-75.

31. Aranda $S$, Jefford $M$, Yates $P$, et al. Impact of a novel nurse-led prechemotherapy education intervention (ChemoEd) on patient distress, symptom burden, and treatment-related information and support needs: results from a randomised, controlled trial. Ann Oncol 2012;23:222-31.

32. Halkett G, Merchant S, Jiwa M, et al. Effective communication and information provision in radiotherapy-the role of radiation therapists. $J$ Radiother Pract 2009;9:3-16.

33. Halkett GK, Schofield P, O'Connor M, et al. Development and pilot testing of a radiation therapist-led educational intervention for breast cancer patients prior to commencing radiotherapy. Asia Pac J Clin Oncol 2012:8:e1-8.

34. Halkett GK, O'Connor M, Aranda S, et al. Pilot randomised controlled trial of a radiation therapist-led educational intervention for breast cancer patients prior to commencing radiotherapy. Support Care Cancer 2013;21:1725-33.

35. Hawkins N, Sanson-Fisher R, Shakeshaft A, et al. The multiple baseline design for evaluating population-based research. Am J Prev Med 2007;33:162-8.
36. Kelly J, Lawrence J, Stevenson Y, et al. Community AIDS/HIV risk reduction: the effects of endorsements by popular people in three cities. Am J Public Health 1992;82:1483-9.

37. Biglan A, Ary D, Wagenaar $C$. The value of interrupted time-series experiences for community intervention research. Prev Sci 2000;1:31-49.

38. West $\mathrm{S}$, Duan $\mathrm{N}$, Pequegnat $\mathrm{W}$, et al. Alternatives to the randomized controlled trial. Am J Public Health 2008;98:1359-66.

39. Wagenaar A, Gehan J, Jones-Webb R, et al. Communities mobilizing for change on alcohol: lessons and results from a 15-community randomized trial. Int J Community Psychol 1999;27:315-26.

40. Eccles M, Grimshaw J, Campbell M, et al. Research designs for studies evaluating the effectiveness of change and improvement strategies. Qual Saf Health Care 2003;12:47-52.

41. Jarlais D, Lyles C, Crepaz N; Trend Group. Improving the reporting quality of nonrandomized evaluations of behavioral and public health interventions. Am J Public Health 2004;94:361-6.

42. Ryan $\mathrm{H}$, Schofield $\mathrm{P}$, Cockburn J, et al. How to recognise and manage psychological distress in cancer patients. Eur J Cancer Care 2005;14:7-15.

43. Halkett G, Kristjanson L. Validity and reliability testing of two instruments to measure breast cancer patients' concerns and information needs relating to radiation therapy. Radiat Oncol 2007;2:43: 25 November 2007

44. Schofield P, Gough K, Ugalde A, et al. Cancer Treatment Survey (CaTS): development and validation of a new instrument to measure patients' preparation for chemotherapy and radiotherapy. Psychooncology 2012;21:307-15.

45. Hawthorne G, Richardson J, Osborne R. The Assessment of Quality of Life (AQoL) instrument: a psychometric measure of health-related quality of life. Qual Life Res 1999;8:209-24.

46. Strong V, Waters R, Hibberd C, et al. Emotional distress in cancer patients: the Edinburgh Cancer Centre symptom study. Br J Cancer 2007;96:868-74.

47. Schofield P, Gough K, Carey M, et al. Cancer Treatment Survey (CaTS): a new instrument to measure patients' preparation for chemotherapy and radiotherapy. Pyschooncology 2010;21:307-15. Under review.

48. Hawthorne G, Richardson J, Day NA. A comparison of the Assessment of Quality of Life (AQoL) with four other generic utility instruments. Ann Med 2001;33:358-70.

49. Hawthorne G, Osborne R. Population norms and meaningful differences for the Assessment of Quality of Life (AQoL) measure. Aust N Z J Public Health 2005;29:136-42.

50. Gysels M, Richardson A, Higginson I. Communication training for professionals who care for patients with cancer: a systematic review of effectiveness. Support Care Cancer 2004;12:692-700.

51. Butow P, Cockburn J, Girgis A, et al. Increasing oncologists' skills in eliciting and responding to emotional cues: evaluation of a communication skills training program. Psychooncology 2008;17:209-18.

52. Pendleton D, Schofield T, Tate $\mathrm{P}$, et al. The consultation: an approach to learning and teaching. Oxford: Oxford University Press, 1982

53. National Breast Cancer Centre. Eliciting and responding to emotional cues. Evidence from the literature and recommended steps. Camperdown, NSW: National Breast Cancer Centre, 2007.

54. Peat J, Mellis C, Williams K, et al. Health science research: a handbook of quantitative methods. London: Sage Publications, 2002.

55. Twisk J. Applied longitudinal data analysis for epidemiology. Cambridge University Press, 2003.

56. Chambers S, Schover L, Halford K, et al. ProsCan for Couples: randomised controlled trial of a couples-based sexuality intervention for men with localised prostate cancer who receive radical prostatectomy. BMC Cancer 2008;8:226

57. Barlow J, Wright C, Sheasby J, et al. Self-management approaches for people with chronic conditions: a review. Patient Educ Couns 2002;48:177-87. 